\title{
A reduced cost implementation of low-rank approximation for anisotropic wave propagation
}

\author{
Luize P. R. Baltazar (Federal University of Pará, Brazil), lury C. S. Araújo (Federal University of Pará, Brazil), Murillo J. S. do \\ Nascimento (Federal University of Pará, Brazil) and Jessé C. Costa (Federal University of Pará, Brazil)
}

\begin{abstract}
Copyright 2019, SBGf - Sociedade Brasileira de Geofísica
This paper was prepared for presentation during the $16^{\text {th }}$ International Congress of the Brazilian Geophysical Society, held in Rio de Janeiro, Brazil, 19 August to 22 August 2019.

Contents of this paper were reviewed by the Technical Committee of the $16^{\text {th }}$ International Congress of The Brazilian Geophysical Society and do not necessarily represent any position of the SBGf, its officers or members. Electronic reproduction or storage of any part of this paper for commercial purposes without the written consent of The Brazilian Geophysical Society is prohibited.
\end{abstract}

\section{Abstract}

The simulation of wavefield propagation in anisotropic media is essential to produce subsurface images. Low-rank approximation allows one to obtain a numerical approximation of wavefield evolution operator that honors the kinematics and approximates amplitudes in heterogeneous anisotropic media. Here we discuss our implementation of low-rank approximation for anisotropic wave propagation in 2D and 3D. The main feature of our investigation is the use of row action methods to considerably reduce storage when computing the low-rank matrix. We validate our implementation in $2 D$ and $3 D$ for $T T I$ and tilted orthorhombic media.

\section{Introdução}

O sucesso da exploração sísmica depende da capacidade de se construir imagens da sub-superfície a partir de registros do campo. A construção destas imagens em meios anisotrópicos apresenta desafios adicionais, em função do alto custo computacional para se simular a propagação de ondas em meios elásticos. Para remediar esta limitação, aproximações pseudo-acústicas têm sido propostas para simular a propagação apenas de ondas $\mathrm{qP}$ a um custo computacional duas ordens de magnitude menor que o cálculo da solução exata do campo de onda de ondas qP e qS elásticos (Alkhalifah, 1998). Porém, a equação de pseudo-acústica apesar de simular corretamente a cinemática da propagação de ondas $\mathrm{qP}$ gera também eventos espúrios, que são fragmentos das ondas S. Estes eventos podem interferir na construção de imagens da subsuperfície, assim sendo, vários algoritmos têm sido propostos para eliminar a presença desses fragmentos (Fletcher et al., 2009; Schleicher and Costa, 2016). Neste resumo usamos equações de ondas pseudo-acústicas para simular o campo de onda $\mathrm{qP}$ em modelos com anisotropia e heterogeneidade, resolvendo o problema de solucionar a equação elástica. Esta abordagem utiliza aproximações de baixo-posto (Song, 2001) para representar a evolução do campo de onda $q P$ em um intervalo de tempo arbitrariamente pequeno (Chen and Liu, 2004). Esta abordagem de Chen and Liu (2004) foi estendida posteriormente para meios anisotrópicos
(Etgen and Brandsberg-Dahl, 2009; Fomel et al., 2013). Em meios anisotrópicos estas representações integrais utilizam aproximações assintóticas para representar o campo de onda qP e utilizam apenas a equação iconal (Cerveny, 2005) para determinar o operador de extrapolação do campo.

\section{Metodologia}

\section{Operador de extrapolação}

Seja a equação de onda acústica-escalar

$$
\nabla^{2} p(\mathbf{x}, t)-\frac{1}{v^{2}(\mathbf{x})} \frac{\partial^{2} p(\mathbf{x}, t)}{\partial t^{2}}=0
$$

conhecendo-se o campo pseudo-acústico em um instante $t_{0}$ em todo o domínio espacial, calcula-se sua transformada de Fourier usando

$$
\hat{p}\left(\mathbf{k}, t_{0}\right)=\frac{1}{(2 \pi)^{3}} \int p\left(\mathbf{x}, t_{0}\right) e^{-i \mathbf{k} \cdot \mathbf{x}} d \mathbf{x}
$$

sendo sintetizado em um instante de tempo futuro $t_{0}+\Delta t$ com a transformada de Fourier inversa

$$
p\left(\mathbf{x}, t_{0}+\Delta t\right)=\int \hat{p}\left(\mathbf{k}, t_{0}\right) e^{i \phi(\mathbf{x}, \mathbf{k}, \Delta t)} d \mathbf{k}
$$

O operador de fase $\phi$ é encontrado quando a equação (3) é substituída na equação de onda acústico-escalar (1), obtendo-se

$$
\phi(\mathbf{x}, \mathbf{k}, \Delta t) \approx \mathbf{k} \cdot \mathbf{x}+v(\mathbf{x}, \mathbf{k})|\mathbf{k}| \Delta t+\ldots
$$

Como se pode ver, o operado de fase é função da velocidade de fase do meio, sendo esta a condição necessária para extrapolar a equação (3) em meios anisotrópicos (Etgen and Brandsberg-Dahl, 2009).

\section{Velocidades de fase}

Nosso trabalho envolve a modelagem do campo compressional pseudo-acústico usando a expressão (3) para atualizar os campos em meios anisotrópicos TTI e ortorrômbico. Para calcular as velocidade de fase desses dois meios, usamos a expressão mostrado por Schoenberg and de Hoop (2000) para meios TI, que pode ser escrita para meios TTI por

$$
\begin{array}{r}
\mathscr{H}(\mathbf{s}) \equiv V^{4}-\left(a_{11}\left(\hat{\mathbf{s}} \cdot \hat{\mathbf{s}}-(\hat{\mathbf{s}} \cdot v)^{2}\right)+a_{33}(\hat{\mathbf{s}} \cdot v)^{2}\right) V^{2}+\left(a_{11} a_{33}\right. \\
\left.-\left(a_{13}+2 a_{55}\right)^{2}\right)\left(\hat{\mathbf{s}} \cdot \hat{\mathbf{s}}-(\hat{\mathbf{s}} \cdot v)^{2}\right)(\hat{\mathbf{s}} \cdot v)^{2}=0,
\end{array}
$$

em que $a_{I J}=C_{I J} / \rho$ indicam os parâmetros elásticos normalizados pela densidade (Musgrave, 1970; Cerveny, 
2005), $v$ é o vetor unitário na direção do eixo de simetria e $\hat{\mathbf{s}}$ é o vetor unitário que indica a direção do vetor vagarosidade. Considerando a aproximação pseudoacústica (Alkhalifah, 1998) em que $a_{55} \rightarrow 0$ mantendose fixa a soma $a_{13}+2 a_{55}$ e utilizando os parâmetros de Thomsen (Thomsen, 1986), pode-se reescrever a expressão para a velocidade de fase na forma:

$$
\begin{array}{r}
\mathscr{H}(\mathbf{s}) \equiv V^{4}-\left(C_{\varepsilon}\left(\hat{\mathbf{s}} \cdot \hat{\mathbf{s}}-(\hat{\mathbf{s}} \cdot \boldsymbol{v})^{2}\right)+C_{0}(\hat{\mathbf{s}} \cdot \boldsymbol{v})^{2}\right) V^{2}+C_{0}\left(C_{\varepsilon}-C_{\delta}\right) \\
\left(\hat{\mathbf{s}} \cdot \hat{\mathbf{s}}-(\hat{\mathbf{s}} \cdot \boldsymbol{v})^{2}\right)(\hat{\mathbf{s}} \cdot \boldsymbol{v})^{2}=0
\end{array}
$$

em que $C_{0} \equiv a_{33}$ indica o quadrado da velocidade de propagação ao longo do eixo de simetria, $C_{\varepsilon} \equiv C_{0}(1+2 \varepsilon)$ e $C_{\delta} \equiv C_{0}(1+2 \delta) ; \varepsilon$ e $\delta$ indicam os parâmetros de Thomsen. A equação (6) é a expressão usada para calcular a velocidade de fase para qualquer direção de propagação em um meio TTI com eixo de simetria na direção $v$. Para o meio ortorrômbico fizemos uso de uma generalização da equação proposta por Schoenberg and de Hoop (2000) para meios ortorrômbicos. A expressão fica

$$
\begin{aligned}
\mathscr{H}(\mathbf{x}, \mathbf{s}) \equiv & \frac{1}{2}\left[V^{4}-\left(a_{11}\left(\hat{\mathbf{s}} \cdot v_{1}\right)^{2}+a_{22}\left(\hat{\mathbf{s}} \cdot v_{2}\right)^{2}+a_{33}\left(\hat{\mathbf{s}} \cdot v_{3}\right)^{2}\right) V^{2}\right. \\
& +\left(a_{11} a_{33}-\left(a_{13}+2 a_{55}\right)^{2}\right)\left(\hat{\mathbf{s}} \cdot v_{1}\right)^{2}\left(\hat{\mathbf{s}} \cdot v_{3}\right)^{2} \\
& +\left(a_{22} a_{33}-\left(a_{23}+2 a_{44}\right)^{2}\right)\left(\hat{\mathbf{s}} \cdot v_{2}\right)^{2}\left(\hat{\mathbf{s}} \cdot v_{3}\right)^{2} \\
& \left.+\left(a_{11} a_{22}-\left(a_{12}+2 a_{66}\right)^{2}\right)\left(\hat{\mathbf{s}} \cdot v_{1}\right)^{2}\left(\hat{\mathbf{s}} \cdot v_{2}\right)^{2}\right]=0
\end{aligned}
$$

$v_{i}$ são os vetores ortonormais que determinam a orientação dos planos de simetria. Introduzindo os parâmetros de anisotropia:

$$
\begin{gathered}
\varepsilon_{11} \equiv \frac{a_{11}-a_{33}}{2 a_{33}} \\
\varepsilon_{22} \equiv \frac{a_{22}-a_{33}}{2 a_{33}} \\
\delta_{13} \equiv \frac{\left(a_{13}+a_{55}\right)^{2}-\left(a_{33}-a_{55}\right)^{2}}{2 a_{33}\left(a_{33}-a_{55}\right)} \\
\delta_{23} \equiv \frac{\left(a_{23}+a_{44}\right)^{2}-\left(a_{33}-a_{44}\right)^{2}}{2 a_{33}\left(a_{33}-a_{44}\right)} \\
\delta_{12} \equiv \frac{\left(a_{12}+a_{66}\right)^{2}-\left(a_{11}-a_{66}\right)^{2}}{2 a_{11}\left(a_{11}-a_{66}\right)} \\
\gamma_{1} \equiv \frac{a_{66}-a_{55}}{2 a_{55}} \\
\gamma_{2} \equiv \frac{a_{66}-a_{44}}{2 a_{44}}
\end{gathered}
$$

Observando que no limite pseudo-acústico em que $a_{44}, a_{55}$ e $a_{66} \rightarrow 0$

$$
\begin{gathered}
a_{13}^{2} \approx a_{33}^{2}\left(1+2 \delta_{13}\right) \\
a_{23}^{2} \approx a_{33}^{2}\left(1+2 \delta_{23}\right) \\
a_{12}^{2} \approx a_{11}^{2}\left(1+2 \delta_{12}\right)=a_{33}^{2}\left(1+2 \varepsilon_{11}\right)^{2}\left(1+2 \delta_{12}\right)
\end{gathered}
$$

substituindo (15), (16) e (17) em (7) e considerando anisotropia fraca, temos uma nova expressão para (7), que é

$$
\begin{aligned}
\mathscr{H}(\mathbf{x}, \mathbf{s}) \equiv & \frac{1}{2}\left\{V^{4}-C_{0}\left[\left(\left(1+2 \varepsilon_{11}\right)\left(\hat{\mathbf{s}} \cdot v_{1}\right)^{2}+\left(1+2 \varepsilon_{22}\right)\left(\hat{\mathbf{s}} \cdot v_{2}\right)^{2}\right.\right.\right. \\
& \left.+\left(\hat{\mathbf{s}} \cdot v_{3}\right)^{2}\right) V^{2}+2 C_{0}\left(\varepsilon_{11}-\delta_{13}\right)\left(\hat{\mathbf{s}} \cdot v_{1}\right)^{2}\left(\hat{\mathbf{s}} \cdot v_{3}\right)^{2} \\
& +2 C_{0}\left(\varepsilon_{22}-\delta_{23}\right)\left(\hat{\mathbf{s}} \cdot v_{2}\right)^{2}\left(\hat{\mathbf{s}} \cdot v_{3}\right)^{2} \\
& \left.\left.+2 C_{0}\left(\varepsilon_{22}-\varepsilon_{11}-\delta_{12}\right)\left(\hat{\mathbf{s}} \cdot v_{1}\right)^{2}\left(\hat{\mathbf{s}} \cdot v_{2}\right)^{2}\right]\right\}=0
\end{aligned}
$$

sendo esta a expressão usada para calcular a velocidade de fase em meios ortorrômbicos.

Introdução à matriz de extrapolação e sua aproximação de baixo-posto

A avaliação numérica do operador de extrapolação (3) na forma de quadratura em cada iteração pode ser representada pelo produto

$$
p_{n}(t+\Delta t)=\Delta k_{1} \Delta k_{2} \sum_{m} W_{n, m} P_{m}(t) e^{i \mathbf{k}_{m} \cdot \mathbf{x}_{n}},
$$

em que $\Delta k_{m}$ é o intervalo de amostragem uniforme ao longo da $m$-ésima componente do vetor número de onda, o arranjo $p_{n}(t) \equiv p\left(\mathbf{x}_{n}, t\right)$ contém o campo de onda amostrado uniformemente no domínio do espaço, $P_{m}(t) \equiv$ $P\left(\mathbf{k}_{m}, t\right)$ contém a amostragem uniforme do campo de onda no domínio de Fourier e $W_{n, m}$ contém a discretização do núcleo do operador de evolução, o qual também é chamada de matriz de extrapolação. Para este último,

$$
W_{n, m}=e^{i V\left(\mathbf{x}_{\mathbf{n}}, \mathbf{k}_{\mathbf{m}}\right)\left|\mathbf{k}_{m}\right| \Delta t} .
$$

e seu armazenamento, mesmo para modelos em 2D, é proibitivo computacionalmente. A aproximação de baixoposto é utilizada para fatorar $W_{n, m}$ na forma

$$
W_{n, m} \approx \sum_{N} \sum_{M} U_{n, N} L_{N, M} V_{M, m}
$$

em que $N<<n$ e $M<<m$. $U$ e $V$ são matrizes ortogonais que são obtidas à partir do algorítmo de decomposição QR e a construção acima sugere que $L$ é uma matriz de baixo-posto. Abaixo discutiremos o algorítmo utilizado para calcular esta aproximação.

À partir dessa decomposição, pode ser obtida a evolução do campo na malha de discretização, a qual pode ser escrita na forma

$$
p_{n}(t+\Delta t)=\sum_{N} U_{n, N} \sum_{M} L_{N, M}\left[\Delta k_{1} \Delta k_{2} \sum_{m} V_{M, m} P_{m}(t) e^{i \mathbf{k}_{m} \cdot \mathbf{x}_{n}}\right] \text {. }
$$

A soma evidenciada entre colchetes na equação acima representa uma transformada inversa de Fourier e pode ser calculada de forma eficiente com algoritmo de transformada de Fourier rápida (FFT) (Press, 2007). Portanto, a avaliação do campo $p_{n}(t+\Delta t)$ requer o cálculo de $M$ transformadas de Fourier. Observando que $P_{m}(t)$ é a transformada de Fourier do campo de onda $p_{n}(t)$, ou seja,

$$
P_{m}=\frac{1}{(2 \pi)^{2}} \Delta x_{1} \Delta x_{2} \sum_{q} p_{q}(t) e^{-i \mathbf{k}_{m} \cdot \mathbf{x}_{q}}
$$

a cada iteração é necessário o computar $(M+1)$ FFTs. O algorítmo para modelagem sísmica usando a aproximação de baixo-posto consiste na aplicação sucessiva da equação (22) durante o intervalo de tempo total em que se deseja simular a propagação do campo de onda. 


\section{Cálculo da matriz de baixo posto}

Para realizar a decomposição baixo-posto (21) e calcular a matriz de baixo-posto $L$, usamos a decomposição QR (Golub and Van Loan, 2012), vinculado à técnica de reconstrução algébrica (ART) Kaczmarz.

Dada uma matriz complexa $\mathbf{A}=\left[A_{n, m}\right]$, com $n \in\left\{1, \ldots, n_{l}\right\}$ e $m \in\left\{1, \ldots, m_{c}\right\}$, sua aproximação de baixo posto é definida pela fatoração

$$
\mathbf{A}_{L}=\mathbf{U} \mathbf{L} \mathbf{V}^{H}
$$

na qual $H$ indica o conjugado da matriz transposta, as colunas de $U_{n, N_{l}}$ e $V_{m, M_{c}}$ são ortonormais, $L_{M, N}$ possui posto completo igual ao $\min M_{c}, N_{l}, M \in\left\{1, \ldots, M_{c}\right\}$ e $N \in$ $\left\{1, \ldots, N_{l}\right\}$; tal que $M_{c}, N_{l}<<m_{c}, n_{l}$ e $\left\|\mathbf{A}-\mathbf{A}_{L}\right\|<\varepsilon$.

O cálculo numérico da aproximação de A consiste primeiramente em determinar as matrizes $\mathbf{U}$ e $\mathbf{V}$. Para calcular essas duas matrizes usou-se a decomposição aplicada sequencialmente ao seu espaço coluna e ao espaço linha.

Utilizamos o algoritmo descrito por Fomel et al. (2013) para determinar $M_{c}$ e $N_{l}$. O algoritmo inicia com uma amostragem aleatória de $M$ colunas de A. Em geral $M \approx$ $3 M_{c}$. Neste momento $M_{c}$ é o posto esperado para o espaço coluna de A, coincidente com o posto de $\mathbf{U}$. Em nossos experimentos numéricos este valor foi tomado como 25 . O posto mínimo, $M_{c}$ é estimado observando o valor absoluto dos pivots durante a decomposição $Q R$. Se a razão entre o maior pivot e o menor for menor que $10^{-5}$ a decomposição QR é interrompida e o valor de $M_{c}$ é igual ao número de pivots computados imediatamente antes que o critério de parada seja satisfeito. Terminado estas iterações obtemos a seguinte aproximação para matriz A

$$
\mathbf{A}_{c}=\mathbf{U}_{c} \mathbf{R}_{c}
$$

em que $\mathbf{U}_{c}$ coincide com as $M_{c}$ primeiras colunas da matriz Q. Um procedimento semelhante é aplicado a $\mathbf{A}^{H}$ o que resulta na aproximação

$$
\mathbf{A}_{c}^{H}=\mathbf{V}_{c}^{H} \mathbf{R}_{c}^{H}
$$

em que $\mathbf{V}_{c}^{H}$ coincide com as $N_{l}$ primeiras colunas da matriz Q.

Determinadas as representações de baixo-posto para os geradores do espaço coluna e do espaço linha $U$ e $V$ de $\mathbf{A}$, resta apenas determinar a matriz de baixo-posto $\mathbf{L}$ na aproximação indicada na equação (24).

Para reduzir o custo de armazenamento para se computar a matrix $\mathbf{L}$ utilizamos o algoritmo de Kaczmarz (Kaczmarz, 1993). Este algoritmo tem usado para solução de sistemas lineares esparsos e de grande porte. Em cada iteração a matriz $\mathbf{L}$ é atualizada a partir de um único valor amostrado aleatoriamente de $A_{n, m}$. Em cada iteração é efetuada o seguinte problema de projeção:

$$
\operatorname{argmin}_{\left\{\mathbf{L}^{k}\right\}} \frac{1}{2}\left(\mathbf{L}^{k}-\mathbf{L}^{k-1}\right)^{H}\left(\mathbf{L}^{k}-\mathbf{L}^{k-1}\right)
$$

com

$$
A_{i, j}=\sum_{m} \sum_{n} U_{i, m} L_{m, n}^{k} V_{n, j}^{*} .
$$

na qual $*$ representa o complexo conjugado.
A função Lagrangeana associada a este problema é

$$
\begin{array}{r}
\mathscr{L}\left(L_{p, q}^{k}, \lambda\right)=\frac{1}{2} \sum_{m} \sum_{n}\left(L_{m, n}^{k}-L_{m, n}^{k-1}\right)^{*}\left(L_{m, n}^{k}-L_{m, n}^{k-1}\right) \\
+\lambda_{i, j}^{*}\left(A_{i, j}-\sum_{m} \sum_{n} U_{i, m} L_{m, n}^{k} V_{n, j}^{*}\right) .
\end{array}
$$

Os pontos extremos são dados pela condição

$$
\frac{\partial \mathscr{L}}{\partial L_{p, q}^{k}}=\left(L_{p, q}^{k}-L_{p, q}^{k}\right)^{*}-\lambda_{i, j}^{*} U_{i, p} V_{q, j}^{*}=0
$$

O que resulta na seguinte expressão para os multiplicadores de Lagrange:

$$
\lambda_{i, j}=\frac{A_{i, j}-\sum_{m} \sum_{n} U_{i, m} L_{m, n}^{k-1} V_{n, j}^{*}}{\left(\sum_{m} U_{i, m} U_{i, m}^{*}\right)\left(\sum_{n} V_{n, j} V_{n, j}^{*}\right)}
$$

e na expressão para atualização da matriz:

$$
L_{p, q}^{k}=L_{p, q}^{k-1}+\lambda_{i, j} U_{i, p}^{*} V_{q, j}
$$

Com o resultado da equação acima, podemos determinar a matriz de baixo-posto de uma matriz $A$ complexa. Aplicamos esta técnica na matriz de extrapolação e fizemos as modelagens que se serão mostradas na próxima seção. O posto da matriz de baixo-posto é indicado em cada resultado numérico.

\section{Resultados}

\section{Meio TTI}

No primeiro teste numérico, a velocidade em relação ao eixo vertical, os parâmetros de Thomsen e os ângulos do eixo de simetria TTI em relação à vertical são mostrados pela Figuras (1a), (1b), (1c) e (1d), respectivamente. Para este modelo o posto estimado foi de $M=15$ e $N=15$ para um um operador de extrapolação com intervalo de evolução de $1 \mathrm{~ms}$.

A Figura (2) mostra o campo de onda imediatamente antes da frente de onda atingir o bloco de alta velocidade. Considerando que as propriedades físicas do meio encaixante variam apenas verticalmente, a complexidade da geometria desta frente de onda se deve a anisotropia e a variação lateral do eixo de simetria. A Figura (3) apresenta o campo de onda após a frente de onda atingir o bloco. Os eventos mais relevantes são a reflexão no topo do bloco e as difrações nos vértices do bloco. Nota-se o caráter fortemente assimétrico do campo refletido, o que reitera que o uso modelos anisotrópicos é indispensável para o correto posicionamento dos eventos nas imagens sísmicas quando o meio apresenta anisotropia. A simulação da propagação exclusivamente de ondas $\mathrm{qP}$ neste modelo usando algoritmos para solução da equação pseudo-acústica (Alkhalifah, 1998) produziria instabilidade (Fletcher et al., 2009). 


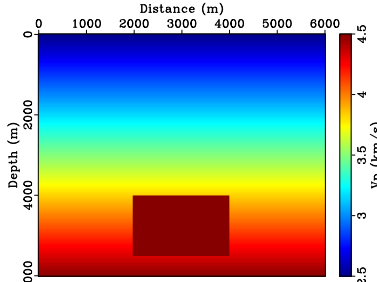

(a)
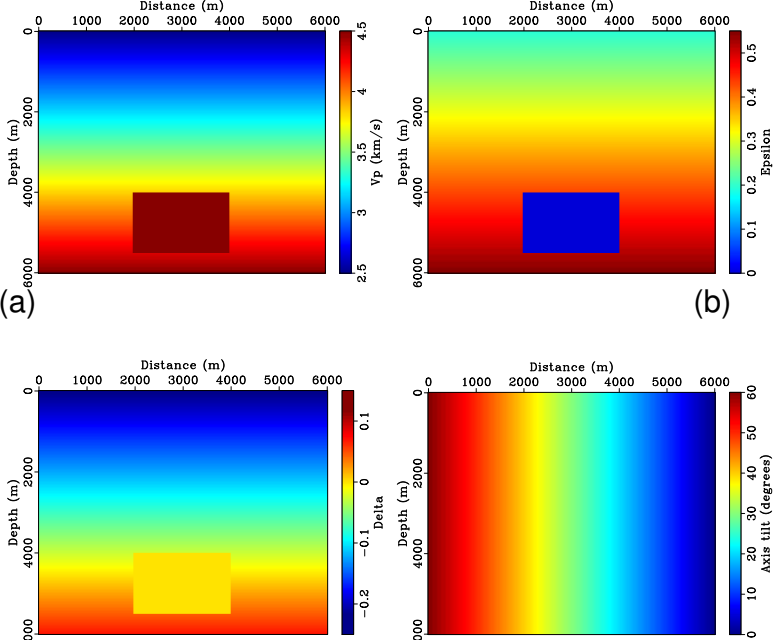

(c)

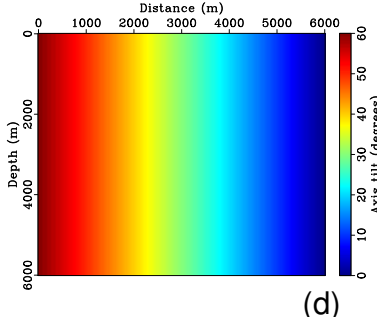

Figura 1

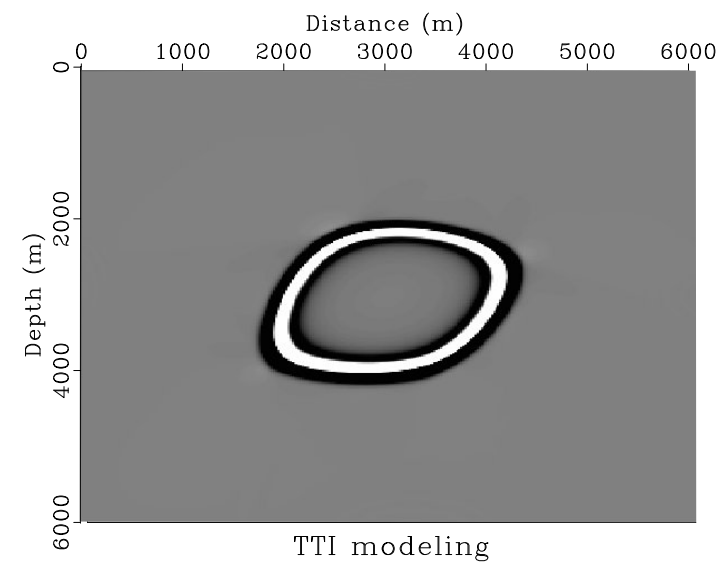

Figura 2: Snapshot do campo de onda qP propagado no modelo do bloco.

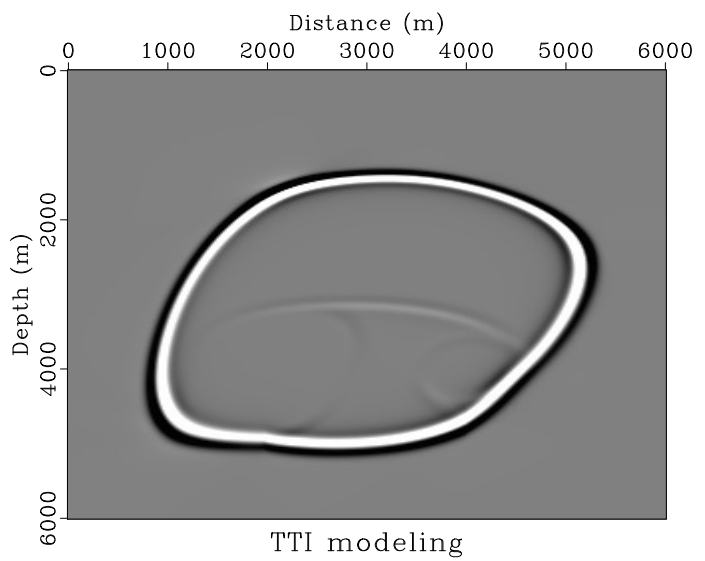

Figura 3: Snapshot do campo de onda qP propagado no modelo do bloco.

No segundo teste numérico, a velocidade em relação ao eixo vertical, o parâmetros de Thomsen e os ângulos do eixo de simetria TTI em relação à vertical são mostrados pelas Figuras (4a), (4b), (4c) e (4d), respectivamente. Para este modelo o posto estimado foi de $M=11$ e $N=11$ para um um operador de extrapolação com intervalo de evolução de 1,333333 m.

Este modelo foi construído pela empresa Britsh Petroleum (Billette and Brandsberg-Dahl, 2005) e divulgado para servir de benchmark na avaliação de algoritmos de modelagem e imageamento na presença de anisotropia. O modelo representa uma região estruturalmente complexa devido as deformações produzidas nos estratos sedimentares produzidas por tectônica salina. Adicionalmente há forte anisotropia em algumas camadas, especificamente, na camada sobrejacente a camada de sal.

O resultado da simulação numérica para os instantâneos do campo de onda qP está apresentado nas Figuras (9) e (10). A complexidade do campo de onda visto nesta figura é decorrente do espalhamento múltiplo sofrido pelo campo de onda. Este efeito é bem pronunciado no diápiro, que produz reflexões múltiplas e difrações. Em consequência há uma marcante variação na iluminação produzida pelo campo de onda qP em subsuperfície. A Figura (9) mostra a variação de iluminação entre as regiões a esquerda e a direita do domo salino. A Figura (10) que apresenta o campo de onda em um instante posterior mostra o efeito das reverberações sofridas pelo campo de onda dentro do corpo de sal. A presença de forte variação na iluminação sísmica e o espalhamento múltiplo do campo de onda pelo diápiro são as duas principais causas para a perda de qualidade nas imagens sísmicas nas regiões sub-sal.

(a)

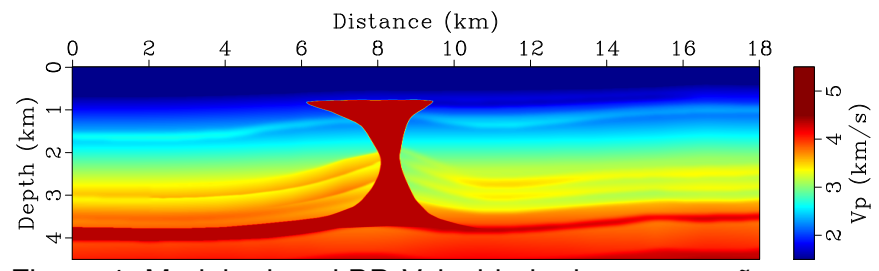

Figura 4: Modelo de sal BP. Velocidade de propagação ao longo do eixo de simetria em $\mathrm{km} / \mathrm{s}$.

(b)

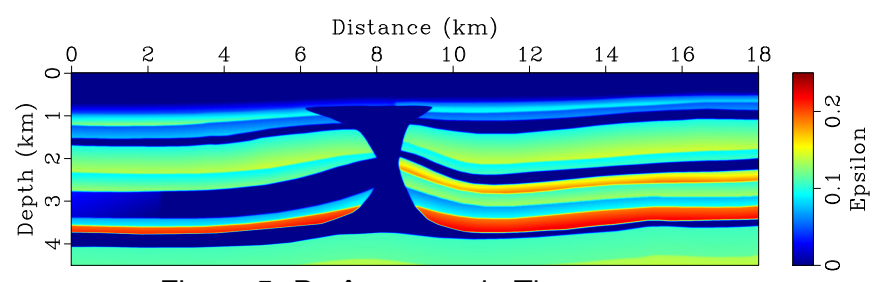

Figura 5: Parâmetro $\varepsilon$ de Thomsen.

\section{Meio ortorrômbico}

Para o terceiro teste foi considerado o meio ortorrômbico homogêneo com os parâmetros de anisotropia dados por $\varepsilon_{11}=0.24, \varepsilon_{22}=0.24, \delta_{13}=0.1, \delta_{23}=0.1, \delta_{12}=0.1 \mathrm{e}$ $C_{0}=4 \mathrm{~km}^{2} / \mathrm{s}^{2}$. A direção dos eixos de orientação foram 
(c)

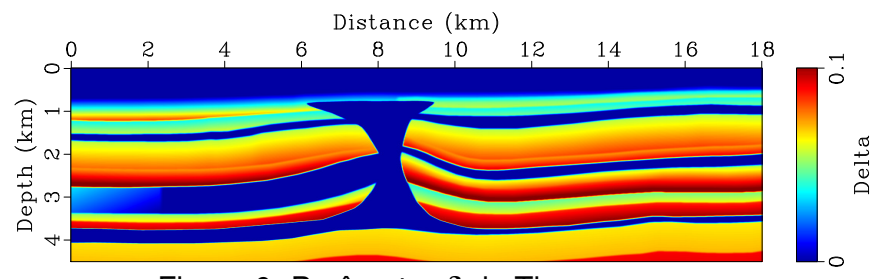

Figura 6: Parâmetro $\delta$ de Thomsen.

(d)

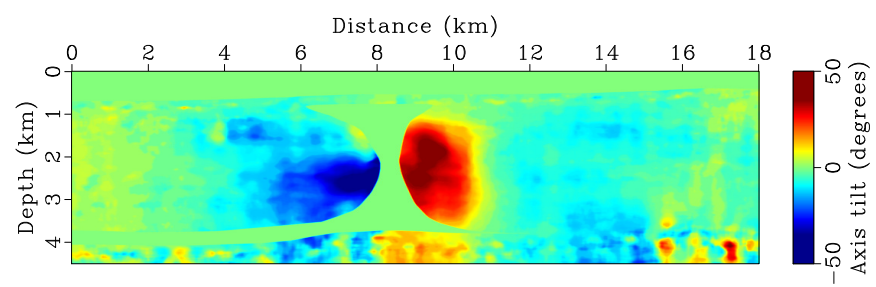

Figura 7: Inclinação do eixo de simetria em graus.

Figura 8: modelos

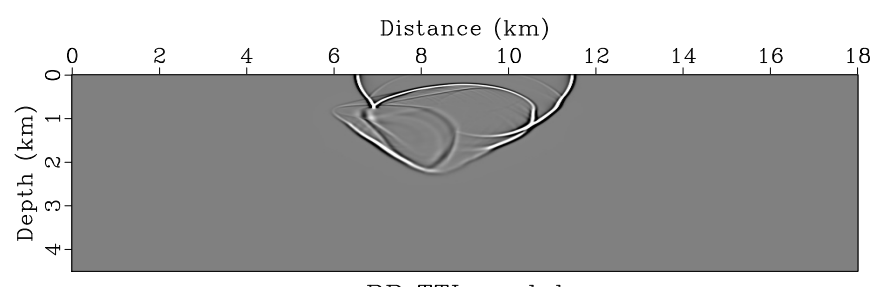

BP TTI model

Figura 9: Snapshot da modelagem no modelo da BP.

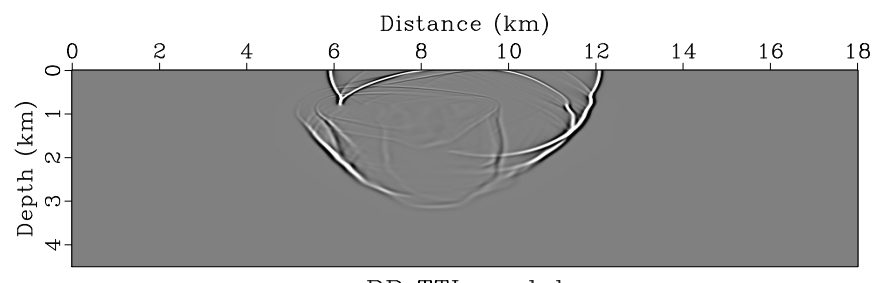

BP TTI model

Figura 10: Snapshot da modelagem no modelo da BP.

$v_{1}=[1,0,0], v_{2}=[0,1,0]$ e $v_{3}=[0,0,1]$. O resultado obtido pode ser visto na Figura (11). Para este modelo o posto estimado foi de $M=2$ e $N=2$.

O próximo resultado é um snapshot da modelagem em um bloco com duas camadas homogêneas com propriedades anisotrópicas diferentes. A camada superior possui como parâmetros $\varepsilon_{11}=\varepsilon_{22}=0.1, \delta_{12}=\delta_{13}=\delta_{23}=0.1$ e $C_{0}=$ $2 \mathrm{~km}^{2} / \mathrm{s}^{2}$, e a camada inferior $\varepsilon_{11}=\varepsilon_{22}=0.2, \delta_{12}=\delta_{13}=$ $\delta_{23}=0.2$ e $C_{0}=4 \mathrm{~km}^{2} / \mathrm{s}^{2}$. A orientação dos planos especulares na primeira camada foi $v_{1}=[1,0,0], v_{2}=$ $[0,1,0]$ e $v_{1}=[0,0,1]$ e da segunda camada $v_{1}=[1,0,0]$, $v_{2}=[0,1,0]$ e $] v_{3}=[0,-0.707,0.707]$.

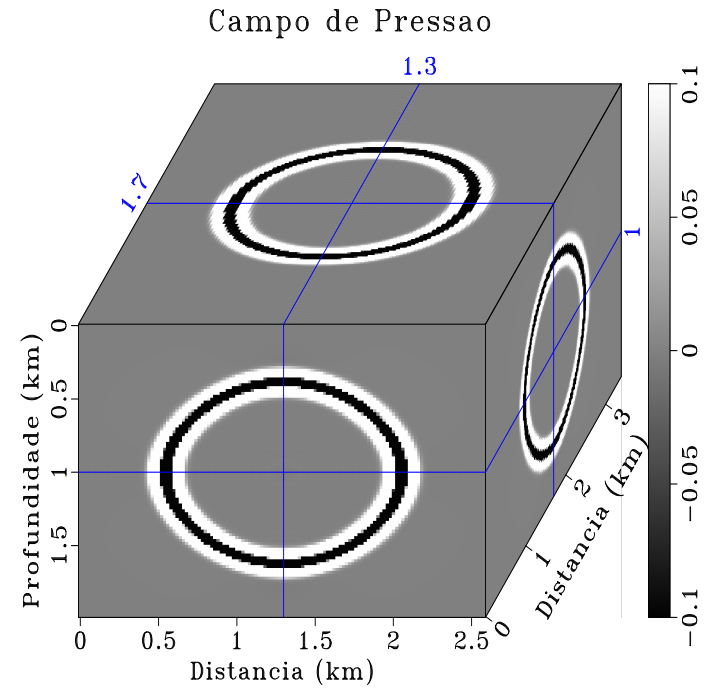

Figura 11: Snapshot do campo de onda em um meio homogêneo anisotrópico ortorrômbico. A fonte sísmica foi colocada exatamento no meio bloco na posição $x=1.3 \mathrm{~km}$, $x=1.0 \mathrm{~km}$ e $y=1.7 \mathrm{~km}$.

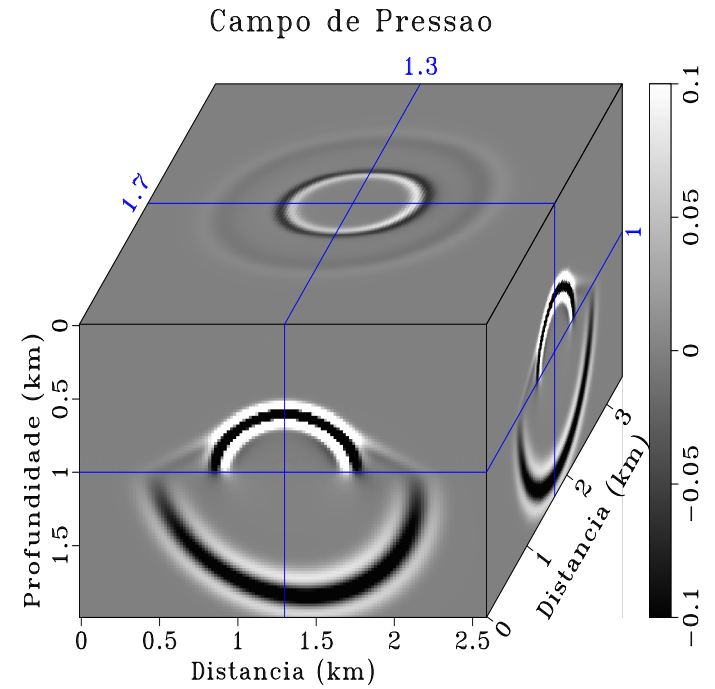

Figura 12: Snapshot da modelagem em um meio heterogêneo. Esse meio é formado por duas camadas anisotrópicas homogêneas. Foi feita uma rotação em torno do eixo $v_{1}$ de 45 graus na camada inferior. A fonte sísmica foi colocada exatamento no meio bloco na posição $x=1.3$ $\mathrm{km}, x=1.0 \mathrm{~km}$ e $y=1.7 \mathrm{~km}$.

\section{Conclusões}

Apresentamos uma implementação do método low-rank com custo de armazenamento reduzido durante o cálculo iterativo da matrix de baixo posto. Adicionalmente, nossos experimentos numéricos indicam que o algoritmo de Kaczmarz tem um custo computacional bastante reduzido quando comparado com o custo da decomposição QR. Finalmente, os instantâneos dos campos de ondas simulados nos meios TTI e ortorrômbicos mostram a robustez e acurácia do algoritmo de baixo-posto para simulação da propagação de ondas qP em meios anisotrópicos heterogêneos. 


\section{Referências}

Alkhalifah, T., 1998, Acoustic approximations for processing in transversely isotropic media: Geophysics, 63, 623-631.

Billette, F. J., and S. Brandsberg-Dahl, 2005, The 2004 bp velocity benchmark: 67th EAGE Conference \& Exhibition, B305.

Cerveny, V., 2005, Seismic ray theory: Cambridge university press.

Chen, J.-B., and H. Liu, 2004, Optimization approximation with separable variables for the one-way wave operator: Geophysical Research Letters, 31.

Etgen, J. T., and S. Brandsberg-Dahl, 2009, The pseudoanalytical method: Application of pseudo-laplacians to acoustic and acoustic anisotropic wave propagation: 79th annual international meeting, seg, expanded abstracts, 2552-2556.

Fletcher, R. P., X. Du, and P. J. Fowler, 2009, Reverse time migration in tilted transversely isotropic (tti) media: Geophysics, 74, WCA179-WCA187.

Fomel, S., L. Ying, and X. Song, 2013, Seismic wave extrapolation using lowrank symbol approximation: Geophysical Prospecting, 61, 526-536.

Golub, G. H., and F. Van Loan, 2012, Matrix computations: JHU Press.

Kaczmarz, S., 1993, Approximate solution of systems of linear equations: International Journal of Control, 57, 1269-1271.

Musgrave, M. J., 1970, Crystal acoustics: Introduction to the study of elastic waves and vibrations in crystals: Holden-Day.

Press, W. H., 2007, Numerical recipes 3rd edition: The art of scientific computing: Cambridge university press.

Schleicher, J., and J. C. Costa, 2016, A separable stronganisotropy approximation for pure qp-wave propagation in transversely isotropic media: Geophysics.

Schoenberg, M., and M. V. de Hoop, 2000, Approximate dispersion relations for $q p-q s v$-waves in transversely isotropic media: Geophysics, 65, 919-933.

Song, J., 2001, The optimized expression of a high dimensional function/manifold in a lower dimensional space: Chinese Scientific Bulletin, 46, 977-984.

Thomsen, L., 1986, Weak elastic anisotropy: Geophysics, 51, 1954-1966. 